- Dental extractions can be safely performed on patients receiving warfarin therapy without stopping or altering the dose of anticoagulant.

- The chances of a thromboembolic attack may be significantly higher than the chance of postoperative bleeding when anticoagulant medication is temporarily stopped.

- Mechanical pressure may be very important and beneficial in stopping postoperative bleeding compared with other alternatives.

\title{
Evaluation of dental extractions, suturing and INR on postoperative bleeding of patients maintained on oral anticoagulant therapy
}

\author{
S. Al-Mubarak, ${ }_{1}^{1}$ N. Al-Ali, ${ }^{2}$ M. Abou Rass, ${ }^{3}$ A. Al-Sohail, ${ }^{4}$ A. Robert ${ }_{1}^{5}$ K. Al-Zoman, ${ }^{6}$ A. Al-Suwyed ${ }^{7}$ \\ and S. Ciancio ${ }^{8}$
}

Objective To examine the consequences of temporary withdrawal of warfarin and/or suturing on bleeding and healing pattern following dental extractions.

Methods Two hundred and fourteen patients on long-term oral anticoagulation (warfarin) therapy scheduled for dental extraction were randomly divided into four groups: no suturing and discontinued (group 1) or continued warfarin (group 2), and suturing and discontinued (group 3) or continued warfarin (group 4). International normalised ratio (INR) was determined at different time points (baseline, days 1, 3 and 7).

Results Discontinuing warfarin reduced INR level significantly at day 1 , which subsequently reached $<1.5$ in 96 out of 104 patients (group 1 and 3). Statistical comparisons among the different treatment groups did not reveal any significant difference regarding bleeding status or healing pattern. Interestingly, patients who received sutures showed higher but insignificant incidence of bleeding postoperatively compared to their respective controls.

Conclusion Dental extractions may be safely performed for patients on anticoagulation therapy provided the INR level is kept $\leq 3.0$ and effective measures of local haemostasis are administered.

1*Dental Department, Sultan Bin Abdulaziz Humanitarian City, Riyadh, Saudi Arabia; ${ }^{2}$ Dental Department, Armed Forces Hospital, Riyadh, Saudi Arabia; ${ }^{3}$ Prince Abdulrahman Bin Abdulaziz Institute for Higher Dental Studies, Riyadh, Saudi Arabia; ${ }^{4}$ Dental Department, Armed Forces Hospital, Riyadh, Saudi Arabia; ${ }^{5}$ Research Center, Sultan Bin Abdulaziz Humanitarian City, Riyadh, Saudi Arabia; ${ }^{6}$ Dental Department, King Faisal Specialist Hospital \& Research Center, Riyadh, Saudi Arabia; ${ }^{7}$ Dental Department, King Abdulaziz Medical City, Riyadh, Saudi Arabia; ${ }^{8}$ Department of Periodontics and Endodontics, School of Dental Medicine, State University of New York at Buffalo, New York, USA

*Correspondence to: Dr Sultan Al-Mubarak, Consultant Periodontist, Chairman of Dental Department, Chairman of Research and Ethics Committee, P.O. Box 64399, Riyadh 11536, Saudi Arabia

Email:smubarak@humanitariancity.org.sa

Online article number E15

Refereed Paper - accepted 5 March 2007

DOI: $10.1038 /$ bdj.2007.725

${ }^{\circledR}$ British Dental Journal 2007; 203: E15
The decision to suture should be made on case-by-case basis, as the trauma associated with soft tissue handling might outweigh its advantages in certain situations like simple extractions.

\section{INTRODUCTION}

Oral anticoagulants are one of the most effective prophylactic/therapeutic medications to combat life-threatening thromboembolic events. ${ }^{1,2}$ The principal adverse effect of oral anticoagulants is haemorrhage that may cause related complications for some patients. ${ }^{3-5}$ Warfarin, a competitive inhibitor of vitamin $\mathrm{K}$, is a commonly prescribed oral anticoagulant to reduce the risk of thromboembolism in patients with mechanical heart valves, deep vein thrombosis and other hypercoagulable states. ${ }^{6}$

The use of the international normalised ratio (INR) has been recommended for monitoring patients' oral anticoagulant therapy. This recommendation is supported by the American College of Chest Physicians, the National Heart, Lung and Blood Institute and the British Society for Haematology. ${ }^{7}$ The INR was developed to incorporate the international sensitivity index (ISI) values and attempt to make prothrombin time (PT) results uniformly useable. The working reference has been calibrated against internationally accepted standard reference preparations which have an ISI value of $1.0 .^{8}$ By definition, those more sensitive to thromboplastin have an ISI of less than 1.0 and those less sensitive are greater than 1.0. The ISI value is critical for calculation of the INR, because the ISI value is the exponent in the formula. Consequently, small errors in the ISI assignment may affect the calculated INR substantially. ${ }^{9}$

Most patients on oral anticoagulant therapy belong to the age group where high prevalence of periodontitis and other dental ailments may necessitate surgical intervention ${ }^{10-13}$ and hence the evaluation of risk of bleeding (with anticoagulation) or thromboembolism (without anticoagulation) is essential. The dilemma of anticoagulant therapy administration before, during and/or after oral surgery continues to prevail, as there are 
Table 1 Description of treatment groups

\begin{tabular}{l|l|l|l|l|l|l}
\hline \multirow{2}{*}{ Study group } & \multicolumn{2}{|l|}{ Treatment } & \multirow{2}{*}{$\begin{array}{l}\text { Age }(\mathrm{y}) \\
\text { mean } \pm \text { SD }\end{array}$} & Gender & \multirow{2}{*}{ Total patients } \\
\cline { 2 - 5 } & Warfarin & Sutures & No & $52.3 \pm 14.3$ & Male & Female \\
\hline$(1)-W-S^{*}$ & No & No & $51.7 \pm 14.7$ & 22 & 26 & 48 \\
\hline$(2)+W-S^{*}$ & Yes & Yes & $48.7 \pm 13.1$ & 27 & 31 & 58 \\
\hline$(3)-W+S^{*}$ & No & Yes & $53.1 \pm 13.7$ & 25 & 31 & 56 \\
\hline$(4)+W+S^{*}$ & Yes & & & 24 & 28 \\
\hline$* W=$ warfarin; $S=$ sutures & & & & & 52 \\
\hline
\end{tabular}

different protocols which have been consistently updated. ${ }^{14,15}$ However, owing to serious complications associated with the withdrawal of anticoagulant therapy, ${ }^{16-18}$ the earlier protocol of temporary discontinuation of anticoagulants for oral surgery ${ }^{19-}$ ${ }^{21}$ has been modified variously to include decreasing the level of anticoagulation, ${ }^{22,23}$ heparin substitution ${ }^{24,25}$ or not altering the anticoagulant regimen..$^{10,11}$

Recent studies have suggested that dental extractions may be carried out with no interruption to the anticoagulant therapy provided local haemostasis is adequately maintained. ${ }^{26-31}$ There are several important modalities for local haemostasis that might be important to minimise postoperative bleeding, including tranexamic acid mouthwash, ${ }^{21,25,26}$ fibrin glue, ${ }^{31-33}$ gelatin sponge, Surgicel and sutures. ${ }^{34,35}$ Blinder et al. ${ }^{27}$ compared the efficacy of various haemostatic methodologies and concluded that gelatin sponge and sutures provide sufficient local haemostasis to perform dental extractions without interruption of oral anticoagulants. Recently, Evans et al. ${ }^{29}$ used polyglactin sutures in combination with oxycellulose dressing for each extraction socket.

The objective of this prospective study was to examine the consequences of temporary withdrawal of warfarin and/or suturing on bleeding and healing pattern following dental extractions.

\section{PATIENTS AND METHODS}

A total of 214 patients who were kept on warfarin as a maintenance dose (2-10 $\mathrm{mg}$ daily) for $>1$ year and required dental extractions were recruited for this study. Patients with history of chronic renal or liver disease or who were on drugs that could affect liver function or haemostasis, other than warfarin, were excluded. Informed consent was obtained from all participants who met the study criteria; they were randomised into four groups (Table 1). Patients in groups 1 and 3 were instructed to stop taking warfarin two days prior to oral surgery and to resume their warfarin treatment 12 hours after dental extractions. This is in accordance with previous studies and suggested by earlier investigators to reduce the INR to a level of 1.5 to $2.0 .^{29,36}$ Patients in groups 2 and 4 continued their prescribed warfarin therapy.

Preoperative blood tests were obtained to measure INR at baseline; INR was also measured on days 1, 3 and 7 postoperatively. Dental extractions were performed with the help of forceps and elevators under local anaesthesia (lidocaine $\mathrm{HCl}$ $2 \%$ and epinephrine 1:100,000); the number of carpules used
Table 2 International normalized ratio (INR) at different time points

\begin{tabular}{|c|c|c|c|c|}
\hline \multirow{2}{*}{$\begin{array}{l}\text { Study } \\
\text { group }\end{array}$} & \multicolumn{4}{|c|}{ INR $($ mean $\pm S D)$} \\
\hline & Pre & Day 1 & Day 3 & Day 7 \\
\hline (1) $-W-S$ & $1.8 \pm 0.4$ & $1.6 \pm 0.4$ & $2.1 \pm 0.7$ & $2.3 \pm 0.6$ \\
\hline$(2)+W-S$ & $2.4 \pm 0.5^{*}$ & $2.4 \pm 0.5^{*}$ & $2.5 \pm 0.7$ & $2.5 \pm 0.6$ \\
\hline (3) $-W+S$ & $1.9 \pm 0.4$ & $1.8 \pm 0.3$ & $1.9 \pm 0.7$ & $2.3 \pm 0.7$ \\
\hline (4) $+W+S$ & $2.7 \pm 0.4^{\# \#}$ & $2.7 \pm 0.6^{\# \#}$ & $2.6 \pm 0.5^{\#}$ & $2.7 \pm 0.6$ \\
\hline
\end{tabular}

was limited to two only for all patients. Postoperative measures to control bleeding were initially applying finger pressure with sterile gauze for 6-10 minutes, then exchanging the used gauze with new sterile gauze when necessary. ${ }^{29,33}$ Patients were asked to keep the gauze on the extraction site for at least 30 minutes and change the gauze with new at the end of their visit. Non-resorbable sutures (UNISILK 4/0, round body noncapillary braided silk black) were used for wound closure for patients in groups 3 and 4. All patients were given detailed written postoperative instructions, the contents of which were verbally illustrated by the treating dentist. Patients were asked to wait for a minimum of one hour within the clinic area; they were closely monitored for bleeding status.

All patients returned for postoperative follow-ups at days 1 , 3 and 7. The status of bleeding and healing were objectively monitored by an independent examiner blinded to the treatment protocol for the four treatment groups. Examiner evaluation was based on healing status and closure of the defect by scar-forming supporting tissue, and on restoration of the form and functions of the damaged area. Complete wound closure by the formation of scar tissue was considered good healing. Incomplete wound closure or the absence of scar tissue formation was considered poor healing. The presence of a solid clot covering the extraction socket was considered as no bleeding, while the presence of a fresh clot that shed easily, or blood oozing was considered as positive bleeding.

Data were analysed using the McNemar test. For categorical correlations Spearman's test was used, whereas $\chi^{2}$ test was 
applied for nonparametric comparisons. P values $<0.05$ were considered as significant.

\section{RESULTS}

Two hundred and fourteen patients were enrolled in the study; the demographic distribution is shown in Table 1. The INR level at baseline and day 1 (after extraction) for group 2 and 4 patients was significantly higher as compared to groups 1 and 3 (Table 2). Resumption of warfarin therapy (groups 1 and 3) gradually increased INR level. However, there was no significant difference at day 7 in INR level among the four study groups (Table 2).

Healing pattern was found to be approximately similar in all treatment groups, showing significant improvement at each consecutive visit. Cross-comparison among the four treatment groups did not show any significant difference in healing at the different postoperative visits (Fig. 1).

Bleeding at postoperative day 1 was not significant; it was slightly higher among patients who continued warfarin therapy $(+\mathrm{W}-\mathrm{S}$ and $+\mathrm{W}+\mathrm{S})$ when compared to the opposite groups $(-\mathrm{W}-\mathrm{S}$ and $-\mathrm{W}+\mathrm{S})$. Furthermore, the improvement continued within all treatment groups and was approximate at day 3 and almost eliminated by the last visit (day 7). Statistical analysis among the various treatment groups did not show any significant difference between the above measures on any of the postoperative visits (Fig. 2). However, abolition of warfarin and suturing (-W-S) appeared to be more favourable with regard to the absence of fresh clots that shed easily or blood oozing frequency in the extraction site. It should be mentioned here that bleeding incidences were of the mild transient type that may be provoked by local aggravating factors, ie chewing on the extraction site.

To examine the relationship between INR value and bleeding incidence, all patients within the four treatment groups were combined together and then categorised into three groups based on INR value (1-2, >2-3 and >3); this was measured relative to the presence or absence of bleeding at the day 1 visit. Bleeding frequency at day 1 postoperatively was significantly less $\left(\chi^{2}=22.65, \mathrm{p}<0.001\right)$ in patients with INR $1-2(13.6 \%)$ or $>2-3(19.8 \%)$ as compared to patients with INR $>3(54.21)$ (Fig. 3). However, bleeding was minimal and not present at the consecutive visits.

The number of teeth extracted in each patient ranged from 1 to 5 . Most of the patients had a single extraction (63.3\%) followed by two teeth (25\%), three teeth (7.5\%), four teeth $(3.3 \%)$ and five teeth $(0.8 \%)$. There was no significant correlation between number of teeth extracted and bleeding status postoperatively.

\section{DISCUSSION}

The incidence of bleeding immediately after dental extractions was relatively higher among groups 2 and 4 as compared to groups 1 and 3. However this effect was not statistically significant (Fig. 2). Evans et al. ${ }^{29}$ also noticed a comparatively higher frequency (although not significant) of bleeding within patients who remained on warfarin therapy compared with controls (26\% versus $14 \%)$. The extraction sites in most of the patients had good closure and scar formation at postoperative follow-ups, irrespective of study groups (Fig. 2). These findings are supported by earlier studies suggesting that anticoagulants

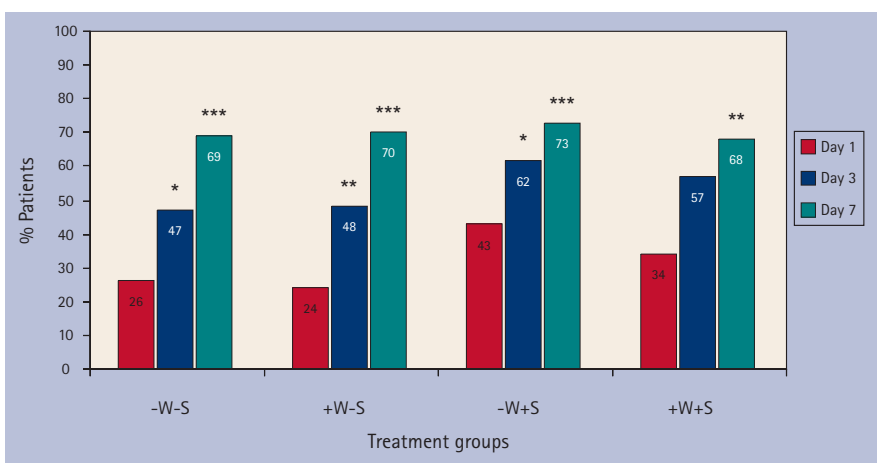

Fig. 1 Wound healing following dental extraction at days 1, 3 and 7 $p$ value versus day 1 (McNemar test); ${ }^{*} p<0.05,{ }^{* *} p<0.01,{ }^{* * *} p<0.001$

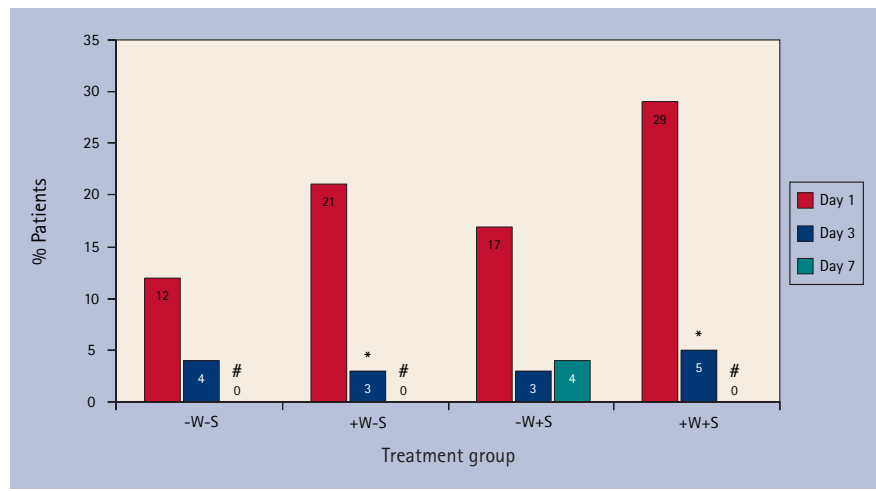

Fig. 2 Postoperative bleeding following dental extraction at days 1, 3 and $7 \mathrm{p}$ value versus day 1 (McNemar test); ${ }^{*} \mathrm{p}<0.05,{ }^{* *} \mathrm{p}<0.01,{ }^{* * *} \mathrm{p}$ $<0.001$. "None of the patients had bleeding (frequency $=0$ )

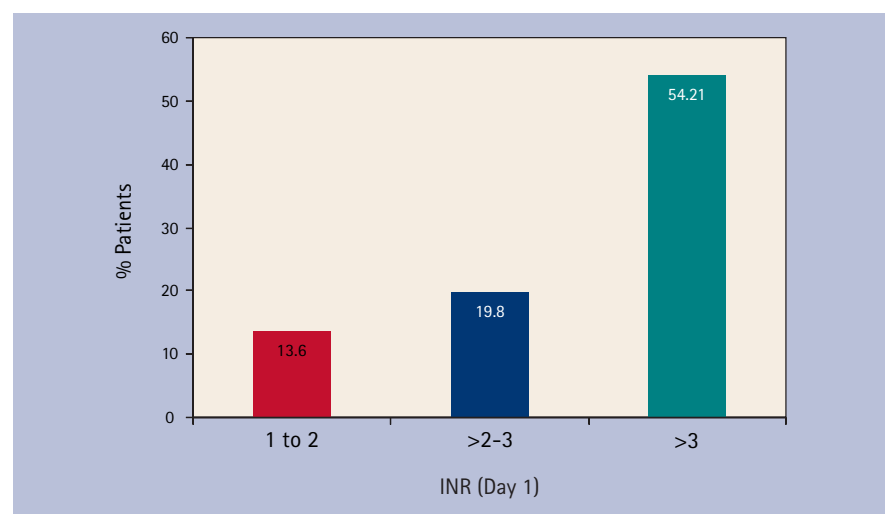

Fig. 3 Categorical presentation of INR (day 1 ) with respect to percentage frequency of patients with bleeding (day 1). Patients with INR $>3$ showed significantly higher frequency of bleeding $\left(\chi^{2}=22.65, p<0.001\right)$

pose no risk of serious bleeding and dental extractions can be carried out safely without stopping warfarin, provided the INR is kept within a safe range..$^{27,29,31,36,37}$ We observed a significant correlation between higher INR level and increased bleeding status. This is in contrast to earlier findings, although it was clinically insignificant. ${ }^{28,38}$ This disparity in results may be attributed to differences in the number of patients and methodology used for surgery and/or local haemostasis. Patients with INR $>3.0$ had significantly higher frequency of bleeding as compared to patients with INR $\leq 3.0$ (Fig. 3), however, the bleeding incidences were of a minimal grade that recovered with time without affecting wound healing. These findings support the notion that in patients with INR $<3.0$, it is relatively safe to perform tooth extraction without a significantly increased risk of bleeding. However, it should be mentioned here that it 
has been reported in other studies that dental extractions can be performed in patients on warfarin therapy with INR up to 4.0 without serious bleeding complications..$^{12,28,39-41}$

Withdrawal of warfarin two days before surgery significantly reduced INR levels before surgery (Table 1) and at day 1 postoperatively, which is in agreement with earlier reports. ${ }^{29,37}$ There was no incidence of thromboembolism even though the INR level in 96 out of 104 patients in groups 1 and 3 was $<1.5$. Devani et al. ${ }^{36}$ had similar observations of INR level $<1.5$ in 14 out of 32 subjects after stopping warfarin for two days. The difficulty in predicting this drop and the associated risk of thromboembolism in warfarin-withdrawal groups outweighs the risk of postoperative bleeding - if any - in patients on maintenance warfarin therapy. ${ }^{36}$

On the other hand, it has been suggested that certain pathological oral microorganisms may cause thromboembolic defects. ${ }^{42,43}$ Data have been obtained that indicate that Streptococcus sanguis interacts with circulating platelets, inducing thromboemboli to cause pulmonary and cardiac abnormalities, and may contribute to the chance of acute thromboembolic events. We tried in this project to avoid the use of any haemostatic agent, ie Surgicel or resorbable sutures, in contrast to previous studies. ${ }^{44-47}$ This was done to minimise the chance of migration of resorbable particles into the blood circulation, which may provoke such a thromboembolic event. It is suggested here that the utilisation of sutures (especially resorbable sutures) may contribute to thromboembolic risk. Together with the discontinuation of warfarin (group 3), this may give an accumulative effect to enhance the occurrence of thromboembolisms. Although this is hypothetical, the combination with the factors mentioned above (discontinuing warfarin in addition to bacterial migration from the newly formed extraction socket to the blood circulation) might exaggerate the occurrence of thromboembolism.

It has been stated and shown in numerous studies that the risks of arterial and venous thromboembolism can be significantly reduced (by up to $80 \%$ ) by anticoagulation therapy. ${ }^{1,48,49}$ Other studies have documented that serious embolic complications including death are three times more likely to occur in patients whose anticoagulant therapy was interrupted, compared to patients with bleeding complications associated with anticoagulation. ${ }^{13}$ In a comparative study comparing the consequences of thromboembolism and postoperative bleeding, it was noticed that approximately $20 \%$ of cases of arterial thromboembolism are fatal and 40\% result in permanent disability, as compared to only $3 \%$ of postoperative bleeding cases resulting in fatality. ${ }^{50}$ Another study showed that an INR $\geq 2.0$ at the time of stroke reduced the incidence and severity of ischaemic stroke, as well as the risk of 30 day mortality from stroke in patients with nonvalvular atrial fibrillation, compared with an INR $<2.0$, aspirin therapy, or no therapy at all..$^{51}$ Therefore, we have to notice here that the temporary discontinuation of warfarin may expose patients to possible risk of thromboembolism equivalent to three days (surgery day, one day pre- and one day post-surgery) without anticoagulation therapy. ${ }^{50}$ This has led to several updates and modifications for some protocols, from stopping anticoagulants before dental extractions ${ }^{50}$ to not altering the maintenance dose, as mentioned by the North West Medical Information Centre, UK. ${ }^{15}$

In this study, there was no reported incidence of postoperative infections. Furthermore, wound healing progressed well in all study groups (Fig. 1). This is supported by earlier studies suggesting that the level of coagulation/anticoagulation has no significant impact on wound healing after dental extractions. ${ }^{11,30,38}$ In this study, the number of teeth extracted per patient ranged from 1-5 and this was not correlated with bleeding tendency. Other investigators also failed to observe any association between postoperative bleeding and number of teeth extracted ${ }^{28-30}$ or complicated extractions. ${ }^{32}$

Our results also showed that suturing played no significant role in bleeding status. Contrary to expectation, group 3 and 4 patients had higher (but insignificant) incidences of bleeding than their respective controls (groups 1 and 2) (Fig. 2). This could be due to the mode of suturing and tissue handling which might complicate tissue healing and wound closure. Therefore, it is suggested here that suturing and invasive manipulation of soft tissue may be avoided in simple extractions and should be administered only when required.

In conclusion, dental extractions can be safely performed in patients on warfarin therapy without stopping or altering the dose of anticoagulant, provided the INR is $\leq 3.0$ and effective local haemostatis has been ensured. Moreover, suturing should be decided on case-by-case basis depending on the extent of surgical trauma and the nature of wound.

This project was funded by King Abdulaziz City for Science and Technology (KACST), Saudi Arabia.

1. Mok C K, Boey J, Wang R et al. Warfarin versus dipyridamole-aspirin and pentoxifylline-aspirin for the prevention of prosthetic heart valve thromboembolism: a prospective randomized clinical trial. Circulation 1985; 72: 1059-1063.

2. Stein P D, Collins J J Jr., Kantrowitz A. Antithrombotic therapy in mechanical and biological prosthetic heart valves and saphenous vein bypass grafts. Chest 1986; 89 (Suppl 2): 46S-53S

3. Owens D E, Calcaterra T C, Aarstad R A. Retropharyngeal hematoma. A complication of therapy with anticoagulants. Arch Otolaryngo/ 1975; 101: 565-568.

4. Lepore M L. Upper airway obstruction induced by warfarin sodium. Arch Otolaryngol 1976; 102: 505-506.

5. Cohen A F, Warman S P. Upper airway obstruction secondary to warfarin-induced sublingual hematoma. Arch Otolaryngol Head Neck Surg 1989; 115: 718-720.

6. Hirsh J, Dalen J E, Deykin D, Poller L. Oral anticoagulants. Mechanism of action, clinical effectiveness, and optimal therapeutic range. Chest 1992; 102 (Suppl 4): 312S-326S

7. Nichols W L, Bowie E J. Standardization of the prothrombin time for monitoring orally administered anticoagulant therapy with use of the international normalized ratio system. Mayo Clin Proc 1993; 68: 897-898.

8. Oertel L B. International normalized ratio (INR): an improved way to monitor oral anticoagulant therapy. Nurse Pract 1995; 20(9): 15-16, 21-22.

9. Florell SR, Rodgers G M 3rd. The PT and the pendulum. Am J Clin Pathol 1996; 106: 699-700.

10. Bailey B M, Fordyce A M. Complications of dental extractions in patients receiving warfarin anticoagulant therapy. A controlled clinical trial. Br Dent J 1983; 155: 308-310.

11. Martinowitz U, Mazar A L, Taicher S et al. Dental extraction for patients on oral anticoagulant therapy. Oral Surg Oral Med Oral Pathol 1990; 70: 274-277.

12. Lippert S, Gutschik E. Views of cardiac-valve prosthesis patients and their dentists on anticoagulation therapy. Scand J Dent Res 1994; 102: 168-171.

13. Wahl M J. Myths of dental surgery in patients receiving anticoagulant therapy J Am Dent Assoc 2000; 131: 77-81.

14. Maidstone Weald Primary Care Trust. Anticoagulation management guidelines folder. Maidstone Weald PCT, 2004. http://www.kentandmedway.nhs.uk/pdf/ Professional_pages/professional_information/Maidstone_Weald/INR_ Protocol L_C.pdf

15. North West Medicines Information Centre. Surgical management of the primary care dental patient on warfarin. Liverpool: North West Medicines Information Centre, 2004.

16. Poller L, Thomson J. Evidence for "rebound" hypercoagulability after stopping anticoagulants. Lancet 1964; 39: 62-64.

17. Duvoisin G E, Brandenburg R O, McGoon D C. Factors affecting thromboembolism associated with prosthetic heart valves. Circulation 1967; 35 (Suppl 4): 170-176.

18. Akbarian M, Austen G, Yurchak P M, Scannell J G. Thromboembolic complications of prosthetic cardiac valves. Circulation 1968; 37: 826-831.

19. Ziffer A M, Scopp I W, Beck J, Baum J, Berger A R. Profound bleeding after dental extractions during dicumarol therapy. N Engl J Med 1957; 256: 351-353. 
20. Scopp I W, Fredrics H. Dental extractions in patients undergoing anticoagulant therapy. Oral Surg Oral Med Oral Pathol 1958; 11: 470-474.

21. Spouge J D. Hemostasis in dentistry, with special reference to hemocoagulation. 3. Principles underlying clinical hemostatic practices in patients with faulty coagulation. Oral Surg Oral Med Oral Pathol 1964; 18: 701-712.

22. Johnson W T, Leary J M. Management of dental patients with bleeding disorders: review and update. Oral Surg Oral Med Oral Pathol 1988; 66: 297-303.

23. DeClerck D, Vinckier F, Vermylen J. Influence of anticoagulation on blood loss following dental extractions. J Dent Res 1992; 71: 387-390.

24. Roser $S$ M, Rosenbloom B. Continued anticoagulation in oral surgery procedures. Oral Surg Oral Med Oral Pathol 1975; 40: 448-457.

25. Johnson-Leong $C$, Rada R E. The use of low-molecular-weight heparins in outpatient oral surgery for patients receiving anticoagulation therapy. J Am Dent Assoc 2002; 133: 1083-1087.

26. Souto J C, Oliver A, Zuazu-Jausoro I, Vives A, Fontcuberta J. Oral surgery in anticoagulated patients without reducing the dose of oral anticoagulant: prospective randomized study. J Oral Maxillofac Surg 1996; 54: 27-32.

27. Blinder D, Manor Y, Martinowitz U, Taicher S, Hashomer T. Dental extractions in patients maintained on continued oral anticoagulant: comparison of local hemostatic modalities. Oral Surg Oral Med Oral Pathol Oral Radiol Endod 1999; 88: 137-140.

28. Blinder D, Manor Y, Martinowitz U, Taicher S. Dental extractions in patients maintained on oral anticoagulant therapy: comparison of INR value with occurrence of postoperative bleeding. Int J Oral Maxillofac Surg 2001; 30: 518-521.

29. Evans I L, Sayers M S, Gibbons A J, Price G, Snooks H, Sugar A W. Can warfarin be continued during dental extraction? Results of a randomized controlled trial. Br J Oral Maxillofac Surg 2002; 40: 248-252.

30. Al-Belasy F A, Amer M Z. Hemostatic effect of n-butyl-2-cyanoacrylate (histoacryl) glue in warfarin-treated patients undergoing oral surgery. J Oral Maxillofac Surg 2003; 61: 1405-1409.

31. Carter G, Goss A, Lloyd J, Tocchetti R. Tranexamic acid mouthwash versus autologous fibrin glue in patients taking warfarin undergoing dental extractions: a randomized prospective clinical study. J Oral Maxillofac Surg 2003; 61: 1432-1435

32. Borea G, Montebugnoli L, Capuzzi P, Magelli C. Tranexamic acid as a mouthwash in anticoagulant-treated patients undergoing oral surgery. An alternative method to discontinuing anticoagulant therapy. Oral Surg Oral Med Oral Pathol 1993; 75: 29-31.

33. Carter G, Goss A. Tranexamic acid mouthwash - a prospective randomized study of a 2-day regimen vs 5-day regimen to prevent postoperative bleeding in anticoagulated patients requiring dental extractions. Int J Oral Maxillofac Surg 2003; 32: 504-507.

34. Anavi Y, Sharon A, Gutman D, Laufer D. Dental extractions during anticoagulant therapy. Refuat Hapeh Vehashinayim 1981; 28: 9-12.

35. Mulligan $\mathrm{R}$, Weitzel K G. Pretreatment management of the patient receiving anticoagulant drugs. J Am Dent Assoc 1988; 117: 479-483.
36. Devani $P$, Lavery K M, Howell C J. Dental extractions in patients on warfarin: is alteration of anticoagulant regime necessary? Br J Oral Maxillofac Surg 1998; 36: 107-111.

37. Gaspar R, Brenner B, Ardekian L, Peled M, Laufer D. Use of tranexamic acid mouthwash to prevent postoperative bleeding in oral surgery patients on oral anticoagulant medication. Quintessence Int 1997; 28: 375-379.

38. Bodner L, Weinstein J M, Baumgarten A K. Efficacy of fibrin sealant in patients on various levels of oral anticoagulant undergoing oral surgery. Oral Surg Oral Med Oral Pathol Oral Radiol Endod 1998; 86: 421-424.

39. Weibert R T. Oral anticoagulant therapy in patients undergoing dental surgery. Clin Pharm 1992; 11: 857-864

40. Campbell J H, Alvarado F, Murray R A. Anticoagulation and minor oral surgery: should the anticoagulation regimen be altered? J Oral Maxillofac Surg 2000; 58: 131-135.

41. Muthukrishnan A, Bishop K. An assessment of the management of patients on warfarin by general dental practitioners in South West Wales. Br Dent J 2003; 195: 567-570.

42. Herzberg M C, Meyer M W. Effects of oral flora on platelets: possible consequences in cardiovascular disease. J Periodontol 1996; 67 (Suppl 10): 1138-1142.

43. Herzberg M C, Weyer M W. Dental plaque, platelets, and cardiovascular diseases. Ann Periodontol 1998; 3: 151-160.

44. Olson R A, Roberts D L, Osbon D B. A comparative study of polylactic acid, Gelfoam, and Surgicel in healing extraction sites. Oral Surg Oral Med Oral Pathol 1982; 53: 441-449.

45. Petersen J K, Krogsgaard J, Nielsen K M, Norgaard E B. A comparison between 2 absorbable hemostatic agents: gelatin sponge (Spongostan) and oxidized regenerated cellulose (Surgicel). Int J Oral Surg 1984; 13: 406-410.

46. Okamoto $\mathrm{G} U$, Duperon D F. Bleeding control after extractions in a patient with aplastic anemia during bone marrow transplantation: report of case. ASDC J Dent Child 1989; 56: 50-55.

47. Matthew I R, Browne R M, Frame J W, Millar B G. Tissue response to a haemostatic alginate wound dressing in tooth extraction sockets. Br J Oral Maxillofac Surg 1993; 31: 165-169.

48. Cannegieter S C, Rosendaal F R, Briet E. Thromboembolic and bleeding complications in patients with mechanical heart valve prostheses. Circulation 1994; 89: $635-641$.

49. Levine M N, Hirsh J, Gent M et al. Optimal duration of oral anticoagulant therapy: a randomized trial comparing four weeks with three months of warfarin in patients with proximal deep vein thrombosis. Thromb Haemost 1995; 74: 606-611.

50. Kearon C, Hirsh J. Management of anticoagulation before and after elective surgery. N Eng/ J Med 1997; 336: 1506-1511.

51. Hylek E M, Go A S, Chang Y et al. Effect of intensity of oral anticoagulation on stroke severity and mortality in atrial fibrillation. N Engl J Med 2003; 349: 1019-1026. 